\title{
Glycerol-silicone elastomers as active matrices with controllable release profiles
}

\author{
Mazurek, Piotr; Brook, Michael A.; Skov, Anne Ladegaard
}

Published in:

Langmuir

Link to article, DOI:

10.1021/acs.langmuir.8b02039

Publication date:

2018

Document Version

Peer reviewed version

Link back to DTU Orbit

Citation (APA):

Mazurek, P., Brook, M. A., \& Skov, A. L. (2018). Glycerol-silicone elastomers as active matrices with controllable release profiles. Langmuir, 34(38), 11559-11566. https://doi.org/10.1021/acs.langmuir.8b02039

\section{General rights}

Copyright and moral rights for the publications made accessible in the public portal are retained by the authors and/or other copyright owners and it is a condition of accessing publications that users recognise and abide by the legal requirements associated with these rights.

- Users may download and print one copy of any publication from the public portal for the purpose of private study or research.

- You may not further distribute the material or use it for any profit-making activity or commercial gain

- You may freely distribute the URL identifying the publication in the public portal

If you believe that this document breaches copyright please contact us providing details, and we will remove access to the work immediately and investigate your claim 


\section{Glycerol-silicone elastomers as active matrices with controllable release profiles \\ Piotr Mazurek, Michael A. Brook, and Anne Ladegaard Skov}

Langmuir, Just Accepted Manuscript • DOI: 10.1021/acs.langmuir.8b02039 • Publication Date (Web): 29 Aug 2018

Downloaded from http://pubs.acs.org on September 3, 2018

\section{Just Accepted}

"Just Accepted" manuscripts have been peer-reviewed and accepted for publication. They are posted online prior to technical editing, formatting for publication and author proofing. The American Chemical Society provides "Just Accepted" as a service to the research community to expedite the dissemination of scientific material as soon as possible after acceptance. "Just Accepted" manuscripts appear in full in PDF format accompanied by an HTML abstract. "Just Accepted" manuscripts have been fully peer reviewed, but should not be considered the official version of record. They are citable by the Digital Object Identifier (DOI®). "Just Accepted" is an optional service offered to authors. Therefore, the "Just Accepted" Web site may not include all articles that will be published in the journal. After a manuscript is technically edited and formatted, it will be removed from the "Just Accepted" Web site and published as an ASAP article. Note that technical editing may introduce minor changes to the manuscript text and/or graphics which could affect content, and all legal disclaimers and ethical guidelines that apply to the journal pertain. ACS cannot be held responsible for errors or consequences arising from the use of information contained in these "Just Accepted" manuscripts. 


\title{
Glycerol-silicone elastomers as active matrices with controllable release profiles
}

\author{
Piotr Mazurek, ${ }^{a}$ Michael A. Brook, ${ }^{b}$ Anne L. Skov ${ }^{a *}$ \\ a Danish Polymer Centre, Department of Chemical and Biochemical Engineering, Technical University of Denmark, \\ DK-2800 Kgs. Lyngby, Denmark. \\ b Department of Chemistry and Chemical Biology, McMaster University, 1280 Main St., W., Hamilton, Ontario, \\ Canada L8S 4M1
}

Keywords: glycerol, silicone, membrane, release, zero-order, absorption, hybrid elastomer

* Corresponding author: al@kt.dtu.dk

\begin{abstract}
Drug release regimes must be controlled for optimal therapeutic effect. While it is relatively straightforward to create first order release matrices, it can be challenging to avoid an initial burst. Matrices with zero-order profiles are perceived to be beneficial in many cases, but are even more difficult to formulate. We describe the straightforward synthesis of elastomeric composites prepared from silicone in which the active is dispersed in glycerol. The release of glycerol-soluble actives from films of these materials was shown to be tunable with respect to the order of release (zero- or firstorder) simply by changing glycerol content. Importantly, release from the elastomers showed no burst effect. The discrete glycerol domains embedded within a silicone matrix act as reservoirs for active
\end{abstract}


substances. Upon contact with aqueous media the active substances are released from matrices exhibiting zero-order, near zero-order or first-order release kinetics. Various parameters that could influence the release process include glycerol content, glycerol domain size or membrane thickness are thoroughly investigated, elucidating guidelines for creating matrices capable of delivering active substances at desired rates. Additionally, the composites proved to absorb significant amounts of liquid water (up to $1850 \%$ of sample mass), a feature that can be tuned by manipulation of the composite structure.

\section{INTRODUCTION}

Increasingly, drug regimens require precise modes of delivery over time; the paradigm of one pill every four hours is frequently not ideal. ${ }^{1-3}$ In the most simple polymer-based drug delivery systems active substances are uniformly dispersed within a polymer in a form of a blend. ${ }^{4,5}$ Such systems typically exhibit first-order release behavior as a consequence of Fickian transport. ${ }^{6}$ This implies that drugs are released relatively rapidly initially, but subsequently the release significantly decelerates. ${ }^{7}$ A wide variety of sophisticated systems based on different polymers have been designed to modify the release profiles. ${ }^{8-12}$ A particularly challenging delivery mode is zero-order, in which the dose of the delivered drug is independent of time or residual concentration in the delivery vehicle. ${ }^{4,13-15}$ In general, zero-order release systems are technologically more difficult to create and therefore result in significantly higher prices. ${ }^{16}$ Sophisticated examples of spherical drug vehicles like porous microspheres or polymer micelles have both proven to be capable of ensuring zero- or near zero-release behavior. ${ }^{17,18}$ Furthermore, various types of gels and matrices have been employed to prepare drug delivery membranes enabling the first-order drug release lasting from few hours up to few weeks and possibly even months. ${ }^{19-21}$ Numerous approaches utilize two or more distinct release 
mechanisms which, upon proper adjustment, result in zero-order release. ${ }^{6,7}$ However, these are not readily prepared or manipulated.

Commercial drug delivery technologies developed so far share some common features. Firstly, all drug delivery systems must be biocompatible and non-toxic. Secondly, the technologies should be simple enough to allow for creating cost-efficient products. We reasoned that an open foam structure could be tailored to give zero-order release, particularly in a topical application, for example wound dressings, where surface area is more important than depth of the device.

We previously described a two-phase glycerol-silicone hybrid elastomer that, depending on formulation, possesses a bicontinuous or closed cell foam structure. Both constituents are understood to be biocompatible and non-toxic in many applications in the biomedical industry. ${ }^{22-25}$ For example, silicone-coated wound dressings are well accepted because the silicone, which is highly porous to oxygen that is needed for healing, does not efficiently adhere to the granulating wound, while providing an excellent seal that prevents bacterial ingress., ${ }^{5,26}$ The glycerol-silicone composites are created simply by providing high shear forces to virtually immiscible mixtures of glycerol and silicone prepolymer. ${ }^{29}$ In this way physically stable glycerol-in-silicone emulsions are formed which, upon cross-linking of the silicone phase, form free-standing two-phase elastomeric composites.

As reported previously, upon contact with an aqueous phase the composite absorbs significant quantities of water and at the same time release glycerol. Glycerol release experiments conducted within 24 hours showed that the percentage amount of released glycerol scales with the glycerol loading ranging from values close to $0 \%$ and $100 \% .^{29}$ It is expected that other substances incorporated into glycerol domains could be released from the matrix in a similar way. That is, the glycerol domains would act as reservoirs for active substances and the drug delivery process would be triggered upon contact with water, including wound exudate. 
Previously some of the basic properties of the glycerol-silicone composites were described, including mechanical properties, biocompatibility, and facile preparation. ${ }^{29,30}$ In this report the main focus is on evaluating properties that are potentially attractive from the perspective of wound care, in particular, water absorption and the capability to release active substances.

\section{EXPERIMENTAL SECTION}

\section{Materials}

A two-component silica-filled hydrosilylation-cured Sylgard 184 silicone kit (S184) was purchased from Dow Corning US. Additional information about Sylgard 184 can be found in the Supporting Information. Hydroquinone (HQ) was purchased from Sigma Aldrich, US. Glycerol was kindly provided by Emmelev A/S, Denmark. All products were used as received.

\section{Sample preparation}

The example provided that leads to a $1.42 \mathrm{~g}$ batch of material may readily be scaled up or down. Using the described method batches between $1 \mathrm{~g}$ and $80 \mathrm{~g}$ were prepared. Hydroquinone $(0.02 \mathrm{~g}, 5$ wt.\%) was dissolved in glycerol $(0.4 \mathrm{~g})$ at $50{ }^{\circ} \mathrm{C}$ using magnetic stirring until a clear solution was obtained (typically around 1-2 hours). Desired amounts of S184 (formed by combining the base 0.909 g and curing agent $0.091 \mathrm{~g}$ in a 10:1 mix following manufacturer's directions, or $0.952 \mathrm{~g}$ of the base and $0.048 \mathrm{~g}$ of the curing agent in a 20:1 mix to give less crosslinked products) and glycerol/HQ (Note: the ratios of the glycerol/silicone products were the same irrespective of the presence of HQ) were mixed at 3500 revolutions per minute (rpm) for 5 min using a dual asymmetric centrifuge SpeedMixer DAC 150 FVZ-K. The obtained glycerol-in-silicone emulsions were cast onto a metal 
mold with $1 \mathrm{~mm}$ thick spacer or coated with various commercial knives in order to obtain films with thicknesses of around $0.1,0.2,0.3,0.4$ or $0.5 \mathrm{~mm}$, respectively. The samples were subsequently cured at $80{ }^{\circ} \mathrm{C}$ for $1 \mathrm{~h}$. Circular disc samples were cut using a custom-made die (25 $\mathrm{mm}$ in diameter and 1 mm thick). Thinner samples were cut out by hand into $4.5 \times 4.5 \mathrm{~cm}$ rectangles using a laboratory knife. Sample nomenclature uses the pattern GX_HQY_S184_Z, where G and X correspond to glycerol and concentration of glycerol in phr (weight parts per hundred weight parts of silicone rubber), HQ and Y correspond to hydroquinone and concentration of hydroquinone dissolved in glycerol (expressed as weight percentage of HQ in glycerol) and S184 corresponds to applied silicone composition, respectively. $\mathrm{Z}$ accounts for varying material parameters that are discussed below. A full description of all investigated samples with corresponding sample names can be found in Table 1 .

Table 1. List of investigated samples with corresponding sample names. The samples are listed chronologically to their occurrence in the manuscript. Compositions based on glycerol, silicone (Sylgard 184 in a 10:1 mix ratio) speed-mixed at $3500 \mathrm{rpm}$ are considered as basic samples. Extensions of the basic compositions are marked bold in the table.

\begin{tabular}{|c|c|c|c|c|}
\hline Sample name ${ }^{a}$ & $\begin{array}{l}\text { Glycerol } \\
\text { content } \\
\text { [phr] }\end{array}$ & $\begin{array}{c}\text { Type and amount } \\
\text { of active } \\
\text { substance in } \\
\text { glycerol } \\
\end{array}$ & $\begin{array}{c}\text { Mixing ratio of } \\
\text { silicone } \\
\text { (base:curing agent) }\end{array}$ & $\begin{array}{c}\text { Composite } \\
\text { morphology b }\end{array}$ \\
\hline S184 & - & - & $10: 1$ & $\mathrm{n} / \mathrm{a}$ \\
\hline G40_S184 & 40 & - & 10:1 & discrete \\
\hline G80_S184 & 80 & - & 10:1 & discrete \\
\hline G120_S184 & 120 & - & $10: 1$ & bicontinuous \\
\hline S184_20:1 & - & - & $20: 1$ & $\mathrm{n} / \mathrm{a}$ \\
\hline G40_S184_20:1 & 40 & - & 20:1 & discrete \\
\hline G80_S184_20:1 & 80 & - & 20:1 & discrete \\
\hline G40_HQ5_S184 & 40 & HQ, 5 wt.\% & $10: 1$ & discrete \\
\hline G80_HQ5_S184 & 80 & HQ, 5 wt.\% & $10: 1$ & discrete \\
\hline G120_HQ5_S184 & 120 & HQ, 5 wt.\% & $10: 1$ & bicontinuous \\
\hline G80_HQ5_S184_2000rpm c & 80 & HQ, 5 wt.\% & $10: 1$ & discrete \\
\hline G80_HQ5_S184_20:1 & 80 & HQ, 5 wt.\% & 20:1 & discrete \\
\hline \multicolumn{5}{|c|}{ additional information on sample thickness is added at the end of each sample name when necessary } \\
\hline iposite morphology dif & ntiates be & en discrete (separ & lycerol domains) and $b$ & continuous morphologies \\
\hline
\end{tabular}


composition mixed at $2000 \mathrm{rpm}$

\section{Methods}

Film thicknesses were measured using an optical microscope Leica DM LB. A FEI Quanta 200 ESEM FEG scanning electron microscope (SEM) was used to investigate the morphologies of composites. Prior to testing, the cross-sections were coated under vacuum conditions and a current of $20 \mathrm{~mA}$ for $5 \mathrm{~s}$ to give a 2 nm-thick gold layer using a high resolution sputter coater Cressington 208HR. Viscosity tests were performed using Ares AR2000x rheometer from TA Instruments equipped with a conical concentric cylinder geometry at a steady state flow. Frequency sweep tests were performed on an Ares G2 rheometer from TA Instruments in order to evaluate viscoelastic properties of elastomers.

In water absorption experiments, the disc samples were immersed in deionized water and the water uptake was monitored gravimetrically over different time intervals. Three samples were tested for each composition and results were averaged. A Keyence VHX-6000 digital microscope was used to examine cross-sections of samples after the water absorption test.

Water droplet absorption was examined using DataPhysics OCA20 goniometer. Droplets (30 $\mu \mathrm{L}$ ) were deposited on investigated films and covered with a glass cuvette to minimize water evaporation. The behavior of a water droplet deposited on pure silicone elastomer was investigated for reference. A video of the water absorption process was recorded and subsequent stages of the water absorption process were evaluated.

Release profiles of HQ from the glycerol-silicone elastomers were determined by immersing composites containing various amounts of glycerol with 5 wt.\% of HQ in deionized water. The progress of HQ release was monitored by measuring changes in concentration of HQ in the aqueous environment. A UV-vis spectrophotometer POLARstar Omega microplate reader by BMG LabTech was used for the tests, and outcomes were compared against a calibration curve for HQ/water 
solutions. Each release profile curve represents an average of three separate experiments. The $1 \mathrm{~mm}$ thick disc samples were tested in tightly sealed conical flasks (placed on a rotary shaker) in order to avoid water evaporation in cases where the measurements lasted for a substantial amount of time. The thinner films $(0.1-0.5 \mathrm{~mm})$ tend to self-adhere when exposed to water therefore they were mounted onto custom-made frames (as presented in Figure 1) to maintain a constant exposed surface area and placed in beakers equipped with magnetic stirring. The water evaporation rate proved to be negligible $(<1.5 \%$ per $8 \mathrm{~h}$ ), therefore, no corrections to the calculations on HQ concentrations were necessary.

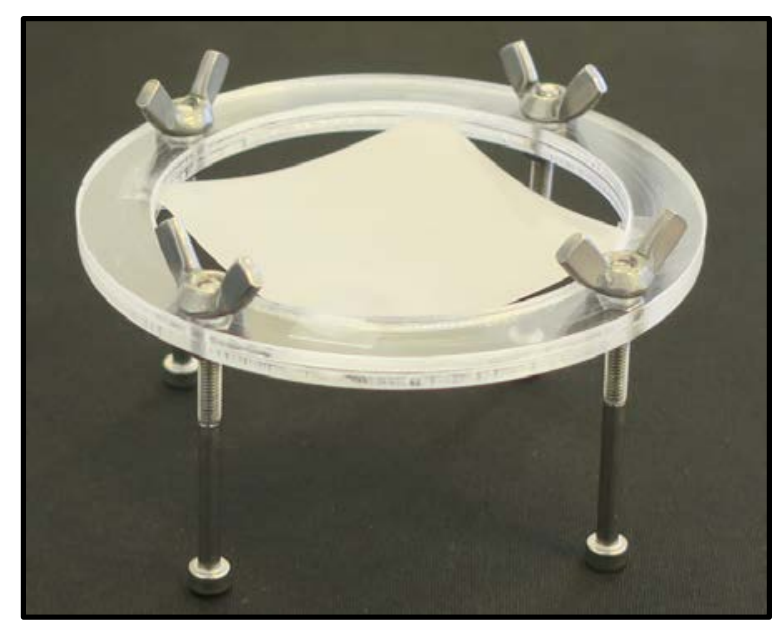

Figure 1. A custom-made frame used for evaluating HQ release rate profiles from thin elastic composites.

\section{RESULTS AND DISCUSSION}

Morphology of the glycerol-silicone composites 
Glycerol-in-silicone emulsions are produced by applying high shear forces to mixtures of glycerol and silicone prepolymer. Upon cross-linking of the silicone phase glycerol-silicone hybrid composites are obtained in which glycerol is embedded in the polymer in the form of discrete droplets as presented in Figure 2A. The study showed that the higher the glycerol loading the more tightly packed the domains are and the thinner the spacing between them becomes. ${ }^{29}$ This holds for the glycerol loadings of up to around $120 \mathrm{phr}$ of glycerol. At this concentration interconnections between the adjacent domains are formed. Intuitively the amount of interconnections scales up as the glycerol loadings increases (a detailed study of the morphologies of samples with high glycerol loading showing the bicontinuous structure is presented in the Supporting Information). As a consequence, a bicontinuous structure is developed, which facilitates faster diffusion of water and other substances throughout the composite, as will be discussed in subsequent sections (Figure 2B).

Furthermore, it was shown that the average droplet diameter decreases slightly with increasing glycerol loading. The average droplet diameters were $3.4 \mu \mathrm{m}, 3.1 \mu \mathrm{m}$ and $2.6 \mu \mathrm{m}$ for samples with 40, 80 and 120 phr of glycerol, respectively. The decline is caused by the sharp increase of viscosity in emulsions with higher content of the dispersed phase. This leads to increasing shear forces that break the dispersed phase into smaller domains. The analysis of average droplet diameter is presented in more detail in the Supporting Information. 

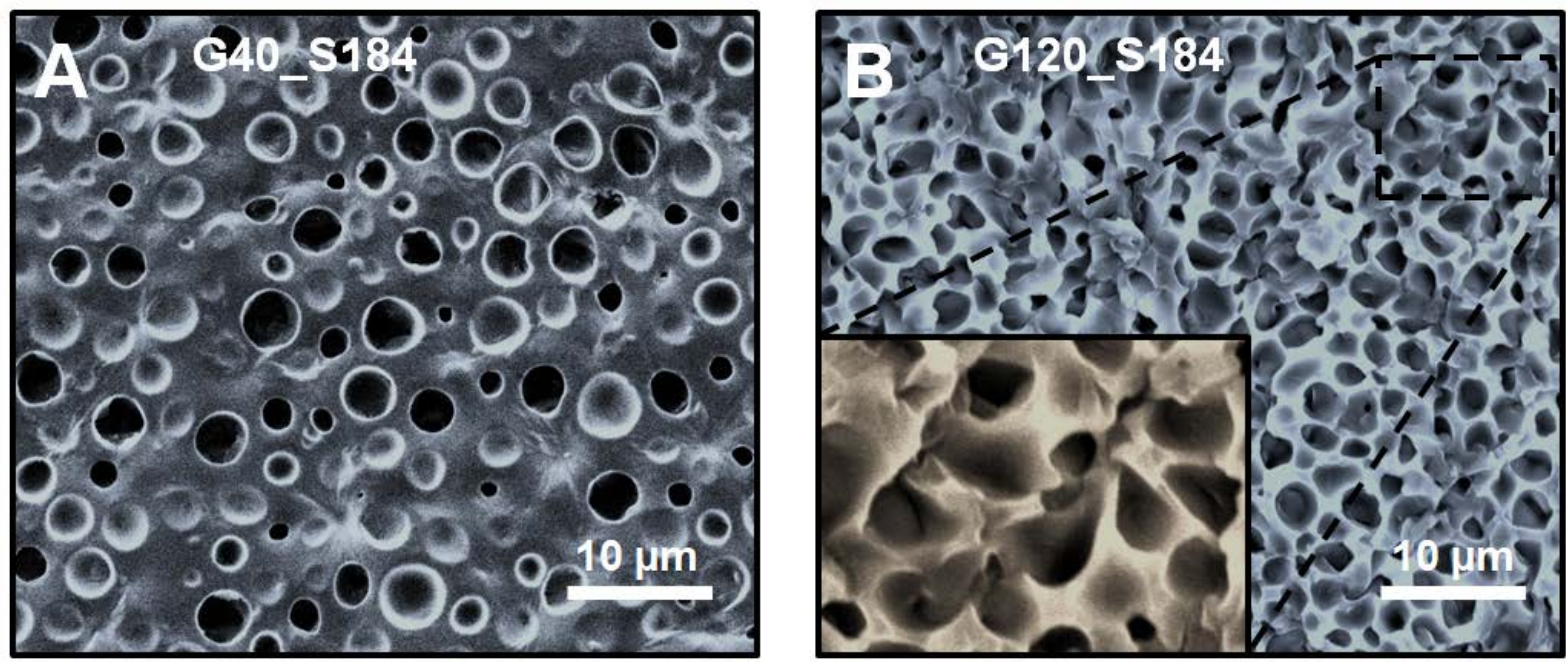

Figure 2. SEM images of composites representing discrete droplet morphology (A) and bicontinuous morphology (B) with interconnections present between the adjacent glycerol domains as illustrated in the enlarged picture in inset.

\section{Water absorption}

As reported previously, glycerol-silicone composites have an ability to absorb water due to the hygroscopic nature of glycerol. ${ }^{29}$ The water absorption is an effect of building up osmotic pressure. As water moves down its osmotic potential gradient, it starts to fill the glycerol domains embedded in the silicone. As was reported by Lee et al., Sylgard 184 (S184) does not swell in the presence of water; therefore, experiments on pure silicone were not conducted. ${ }^{31}$

In this study the S184 silicone kit was mixed in standard ratio 10:1 (base:cross-linker) as recommended by the manufacturer. It is known that the softer the silicone, the more water can potentially be absorbed by the composites, as lower elastic stress has to be overcome in order to expand the glycerol domains. Therefore, elastomer samples based on an off-stoichiometric mixing ratio of 20:1 were also prepared and tested. It is known that by reducing the amount of the crosslinker the cross-linking density will be decreased and the increased fraction of dangling chains will 
lead to an overall drop of the elastic modulus. ${ }^{32}$ The viscoelastic properties of silicone samples mixed in different ratios are presented in Figure 3A. As can be seen, the storage modulus of Sylgard 184 could be successfully decreased from around $100 \mathrm{kPa}$ to around $60 \mathrm{kPa}$ using this method. 
A

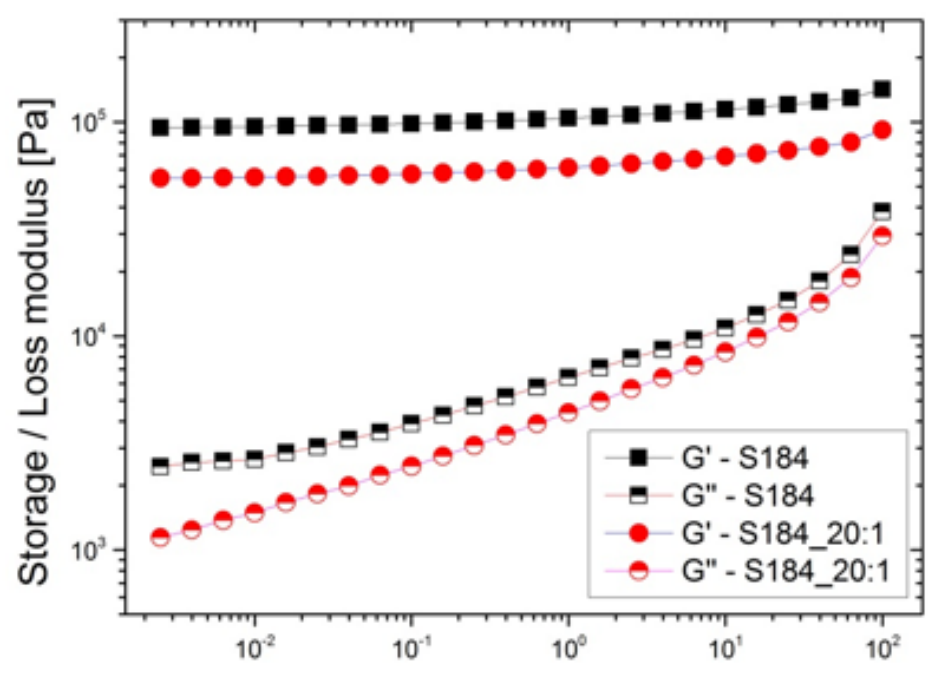

Frequency [Hz]
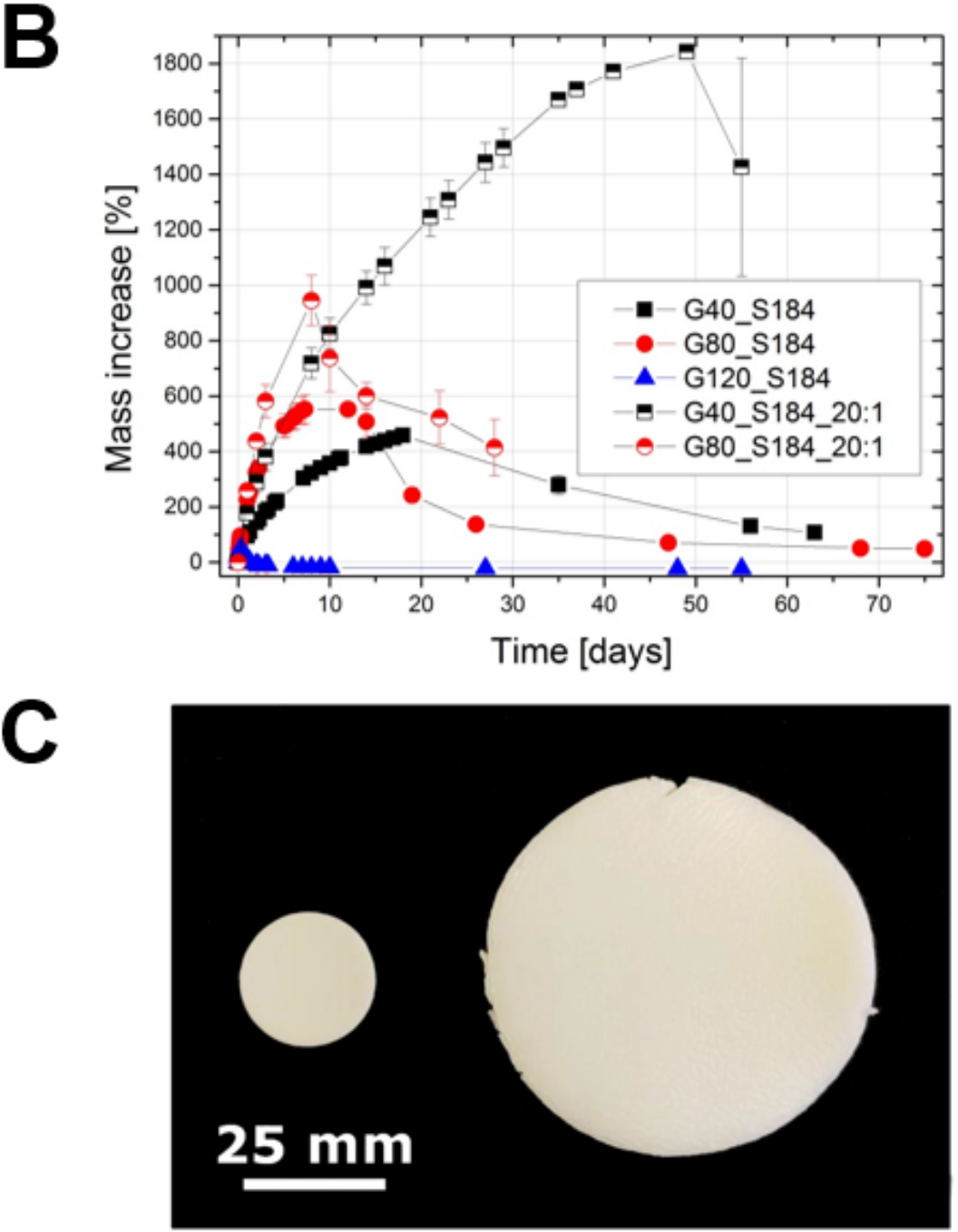
Figure 3. A) Storage and loss moduli of Sylgard 184 mixed in ratios of $10: 1$ and 20:1 (base:crosslinker) measured at room temperature. B) Water absorption profiles of $1 \mathrm{~mm}$ thick disc samples based on different S184 formulations and various glycerol loadings measured at room temperature. C) Sample G40_S184_20:1 before (left) and after (right) the water absorption experiment. The sample mass and diameter increased by $1840 \%$ and $300 \%$, respectively. The samples swelled isotropically and thickness increased approximately $300 \%$ as well.

The long-term water absorption behavior is presented in Figure 3B. The samples G80_S184_10:1 and G80_S184_20:1 absorbed the highest amounts of water at the highest rates for the investigated silicone compositions. The mass of the sample G80_S184_10:1 increased by $550 \%$ after 12 days whereas the mass of the sample G40_S184_10:1 increased by $460 \%$ in 18 days. These two samples have relatively high glycerol loadings, whereby a high osmotic potential gradient is formed. At the same time the discrete glycerol domains are separated by thin silicone domains, and the distance between the glycerol domains and thus the resistance towards expansion scale inversely with the concentration of glycerol. The sample with the highest glycerol content and glycerol content close to the point at which the morphology becomes bicontinuous, G120_S184_10:1, was observed to absorb the lowest amount of water. In addition, the mass of this particular sample started declining after reaching its maximum and the final mass of this particular sample after drying was found to be lower than the initial mass. This verifies that glycerol was washed out during this process due to too low resistance from the silicone matrix between the close adjacent compartments.

Samples with discrete glycerol droplet morphology based on the soft silicone (made using a mixing ratio of base:crosslinker 20:1) absorbed more significant amounts of water at higher rates compared to their harder counterparts, as expected. The sample G80_S184_20:1 absorbed water faster than the sample G40_S184_20:1 due to higher osmotic potential built in the former. However, 
the total mass increase was much more substantial in the case of the sample G40_S184_20:1, which stands in contrast to the compositions based on the S184 mixed in ratio 10:1. The samples G40_S184_20:1 and G80_S184_20:1 absorbed 1840 \% and $940 \%$ of water, respectively. Optical microscopy images of cross-sections of the samples before and after the water absorption experiment are presented in Figure 4. As expected, along with water absorption all samples increased in volume as shown in Figure 3C, where images of a sample G40_S184_20:1 before and after the water absorption experiment are presented. The sample increased in diameter by almost $300 \%$ while retaining favorable elastic properties. However, in all cases the interruption in mass increase, manifested by reaching a maximum sample mass followed by a mass decrease, was related to deterioration of mechanical properties. There is a water absorption limit for each glycerol-silicone silicone composition above which the increasing stress in the silicone phase becomes too high and the material breaks. It is manifested by formation of multiple cracks in the samples, i.e., the material self-destructs. 

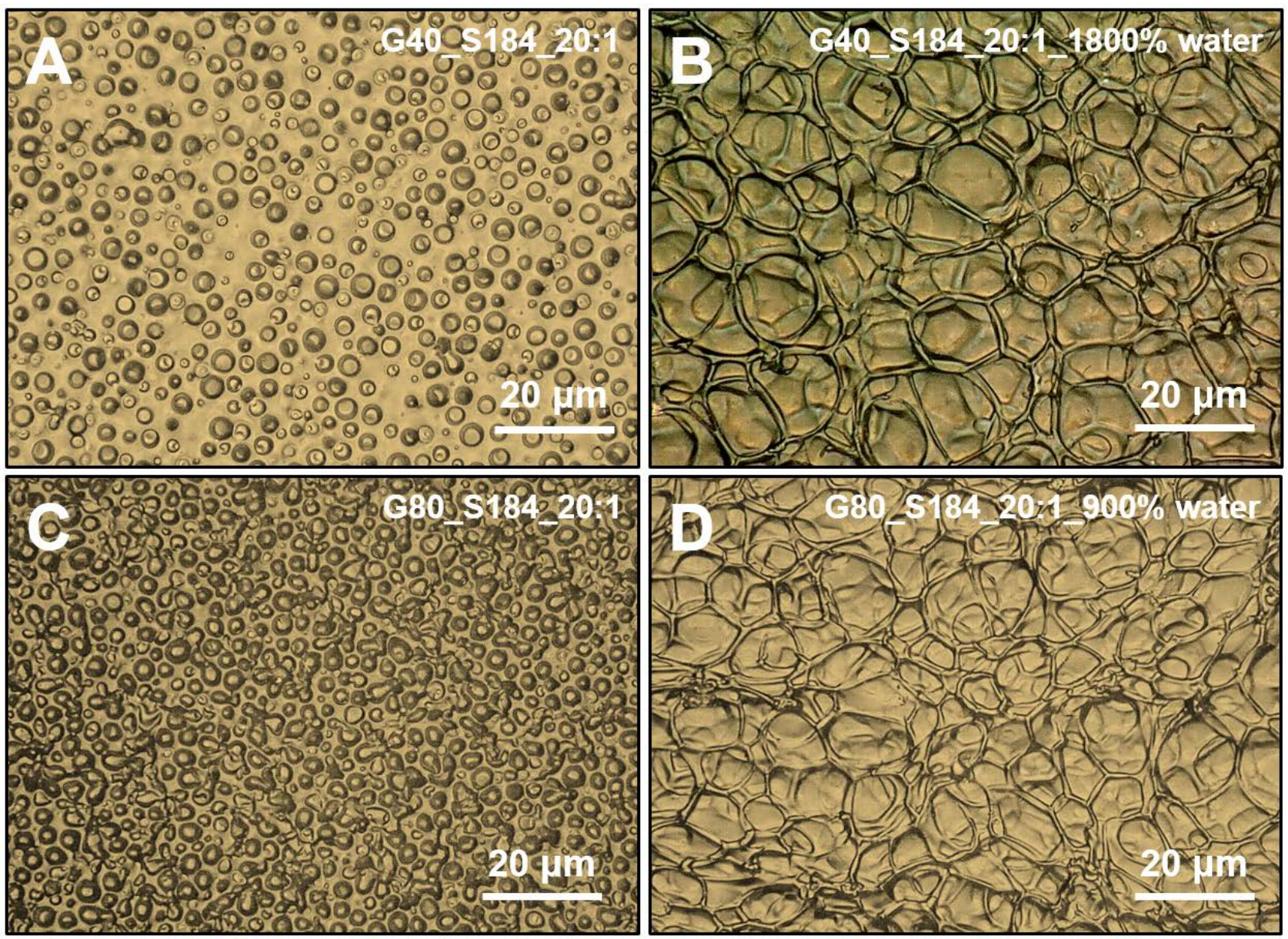

Figure 4. Optical microscopy images of samples G40_S184_20:1 (A and B) and G80_S184_20:1 (C and D) before and after the water absorption experiment.

\section{Water droplet absorption}

Water droplet absorption experiments were conducted in order to better understand and evaluate the processes occurring when different glycerol-silicone composites initially come in contact with water. As can be seen in Figure 5 the droplet dispensed on pure S184 was not absorbed by the elastomer as the sample volume remained unaffected. Furthermore the droplet volume also remained intact proving that the cuvette placed over the droplet efficiently minimized water evaporation. At room temperature and standard atmospheric pressure $30 \mu \mathrm{L}$ water droplets evaporate in around 1-2 
hours. As expected, samples with higher glycerol loadings tend to absorb water faster, in good agreement with observations from the previous section. Interestingly, water taken up by samples G40_S184 and G80_S184 remains in glycerol domains located immediately beneath the water droplet, as shown by deformation of the elastomer, whereas water absorbed by the sample G120_S184 is immediately transported to subsequent glycerol domains. This phenomenon is facilitated by the presence of glycerol interconnections in the composite structure that allows for rapid distribution of absorbed liquid within the composite.

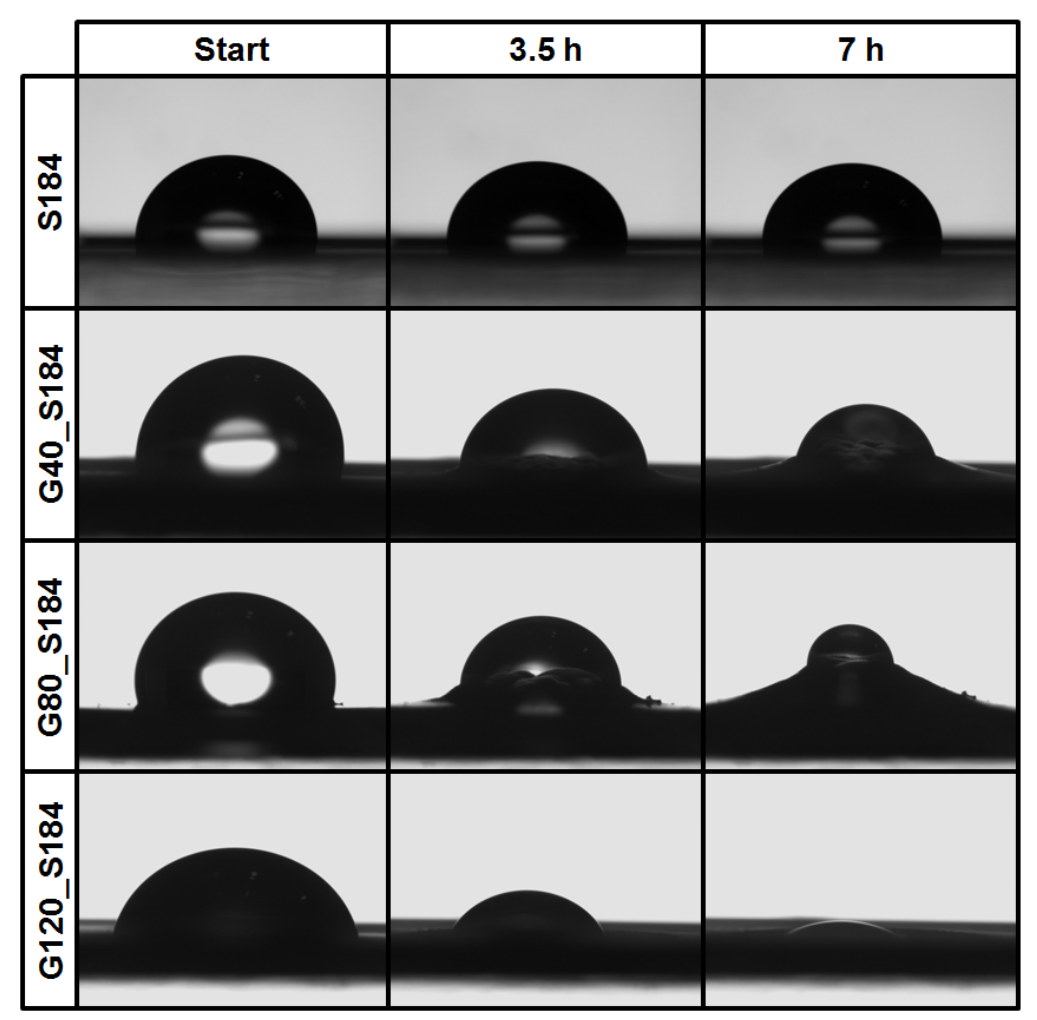

Figure 5. Progress of the water droplet absorption experiment at different times for 4 different compositions. Water absorption rates increase with increasing glycerol loading in the composites. The bicontinuous structure in the sample G120_S184 allows for relatively fast water transportation to the bulk of the material, which stands in contrast to samples with lower glycerol loadings (e.g., 
G80_S184) that tend to accumulate water in glycerol domains immediately underneath the water droplet.

\section{Active substance release}

Hydroquinone was used in this study as a model compound of small size compared to traditional drugs- an easily "traceable” active substance - to investigate release profiles from the glycerolsilicone elastomers. The experiments were conducted until no further increase in HQ concentration in the external water phase was observed. The values obtained from plateau regions of the release profiles were considered to correspond to the full release of HQ from the glycerol-silicone composites. Maximum releases of, on average, $93 \%$ ( $\pm 4 \%$ ) of the theoretical value based on mass of incorporated HQ, glycerol loading and measured film thickness were achieved.

The water absorption study indicates indirectly that there are several factors influencing release rates of substances from the composites. The results of experiments presented in this section are meant to provide an overview of different parameters that influence the release rather than to suggest optimization of ultimate matrix properties, as it is believed that the technology allows for the preparation of numerous products with various properties that can be adapted for different applications.

Influence of glycerol loading

Results presented in Figure 6 indicate that substances are released faster from composites with higher glycerol loadings, which is in a good agreement with findings from the water absorption experiments published previously. ${ }^{29}$ The samples G40_HQ5_S184_1mm, G80_HQ5_S184_1mm and G120_HQ5_S184_1mm release 100 \% of incorporated HQ after around 1, 2 and 7 days, 
respectively. Interestingly, the sample G120_HQ5_S184_1mm released HQ at a constant rate exhibiting a zero-order release profile. It is believed that this behavior is facilitated by the existence of interconnected glycerol channels. Active materials with zero-order or near zero-order release profiles are of great interest, as they allow the delivery of drugs without the common 'burst effect' in which a significant amount of an active substance is released in the initial stage of the process. ${ }^{6,7,33}$ This is a significant advantage over the conventional first- and second-order drug delivery systems as they allow for a full control over the release process. ${ }^{34,35}$

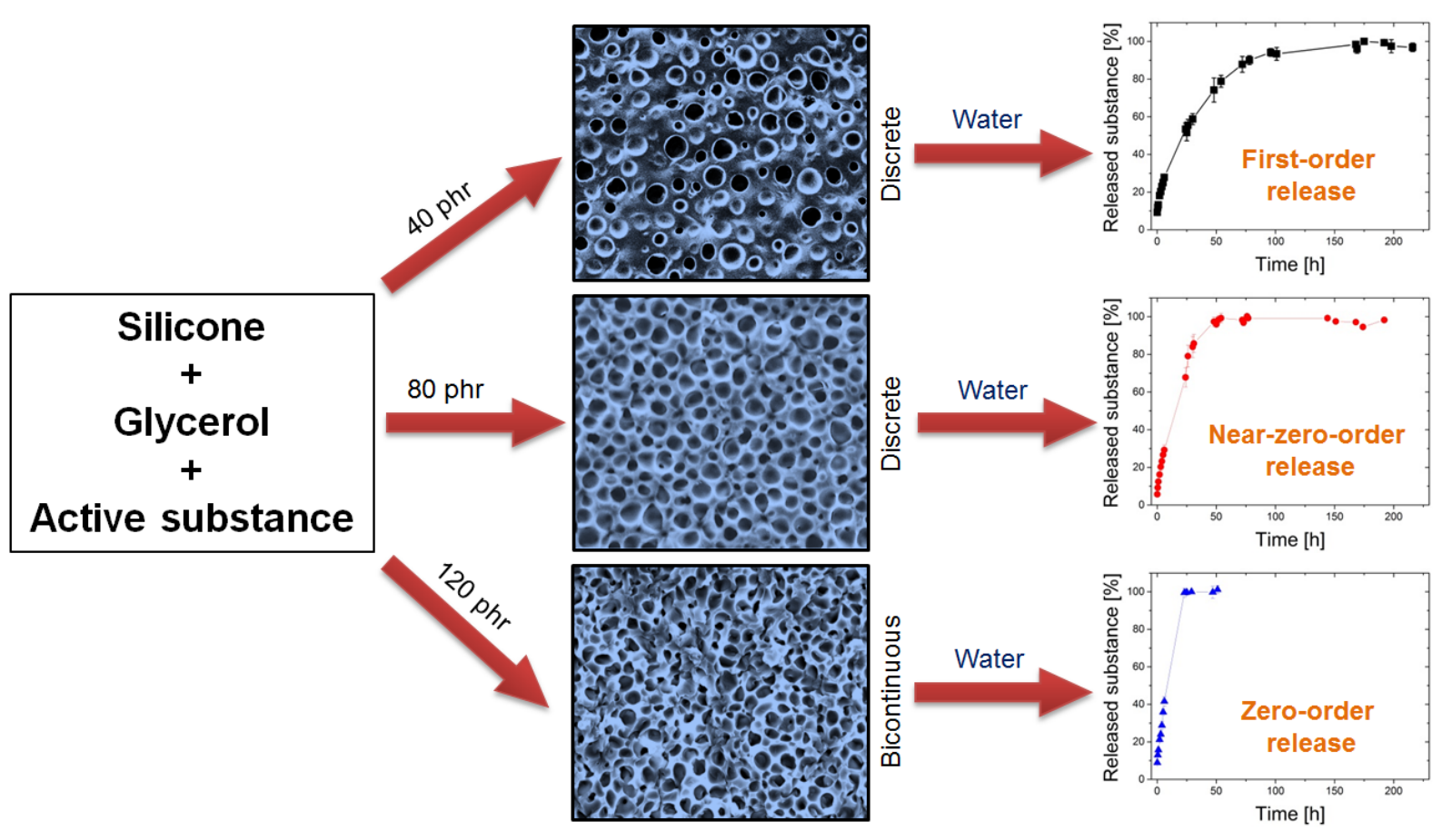

Figure 6. Influence of glycerol loading on the morphology and release kinetics of the glycerolsilicone elastomers. The release profiles from various $1 \mathrm{~mm}$ thick glycerol-silicone elastomers are presented. By increasing the glycerol content a transition from a discrete droplet morphology to a bicontinuous structure can be observed. Upon contact with aqueous media the composites release active substances with different modes varying between first-order release and zero-order release. 
The release rates were shown to vary as a function of the surface area/thickness ratio. For example, release profiles analogous to those of the $1 \mathrm{~mm}$ thick samples were observed in the case of $0.1 \mathrm{~mm}$ composite films (Figure 7A). The complete release of HQ from the sample G80_HQ5_S184_0.1mm was reached after around 2-2.5 hours whereas the 10 times thicker sample needed as much as around 50 hours to release the whole content of HQ.
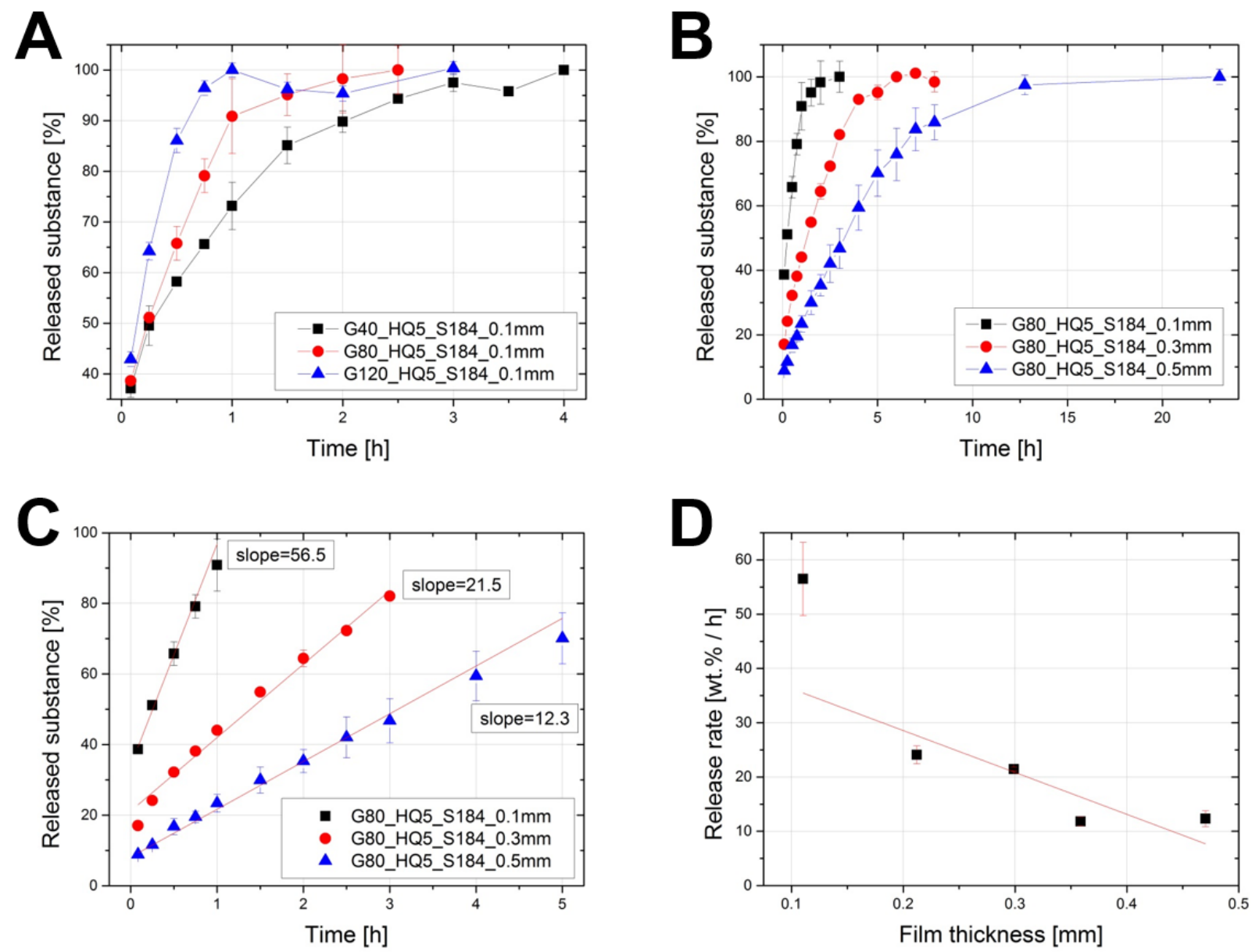

Figure 7. A) Hydroquinone release profiles from various $0.1 \mathrm{~mm}$ thick glycerol-silicone elastomers.

B) Hydroquinone release profiles from glycerol-silicone elastomers of various thicknesses and constant glycerol loading. C) Comparison of release rates (slopes) of samples with $80 \mathrm{phr}$ of glycerol and different thicknesses. D) Dependence of the release rate on the film thickness for compositions G80_HQ5_S184. 
In Figure 7B release data for different composite thicknesses at constant glycerol loading is shown. The data clearly shows that the release profiles of the investigated composites exhibit strong dependence on composite thickness. The entire content of HQ was depleted after around 3, 7 and 23 hours from $0.1,0.3$ and $0.5 \mathrm{~mm}$ thick films, respectively, suggesting a non-linear dependence of release time and film thickness, which is typical for non-zero-order release processes. However, it can be realized that the samples released HQ with constant rates in the first stage of the release process (up to $70-90 \%$ ) indicating existence of near zero-order release kinetics. The release rates were estimated by calculating slopes of the curves as presented in Figure 7C. The release rates were fitted against thicknesses (see Figure 7D) presenting almost linear dependence indicating near zero-order release kinetics.

Influence of glycerol domain sizes on release profiles

As discussed previously, the glycerol domain size can be tuned via controlling the applied shear rate when preparing glycerol-in-silicone emulsions. ${ }^{29}$ Release rates from composites prepared with two different mixing speeds are compared to demonstrate this. A composition with 80 phr of glycerol was exposed to mixing at 2000 rpm or $3500 \mathrm{rpm}$ for two minutes resulting in formation of composites with average glycerol droplet diameters of $5.9 \mu \mathrm{m}$ and $2.5 \mu \mathrm{m}$, respectively. SEM images of the composites are presented in Figure 8. It was expected that the droplet size might influence the release of HQ as morphologies of the two discussed composites differ significantly. However, as can be seen in Figure 9A, the release profiles of the investigated samples were almost identical despite the difference in overall glycerol-silicone interphase area in the samples. This implies that the osmotic potential is the main factor that influenced the release in these systems. Consequently, it can be 
deducted that lower shear forces can be applied in order to produce emulsions that become a basis for creating elastomer matrices exhibiting desired release behavior.
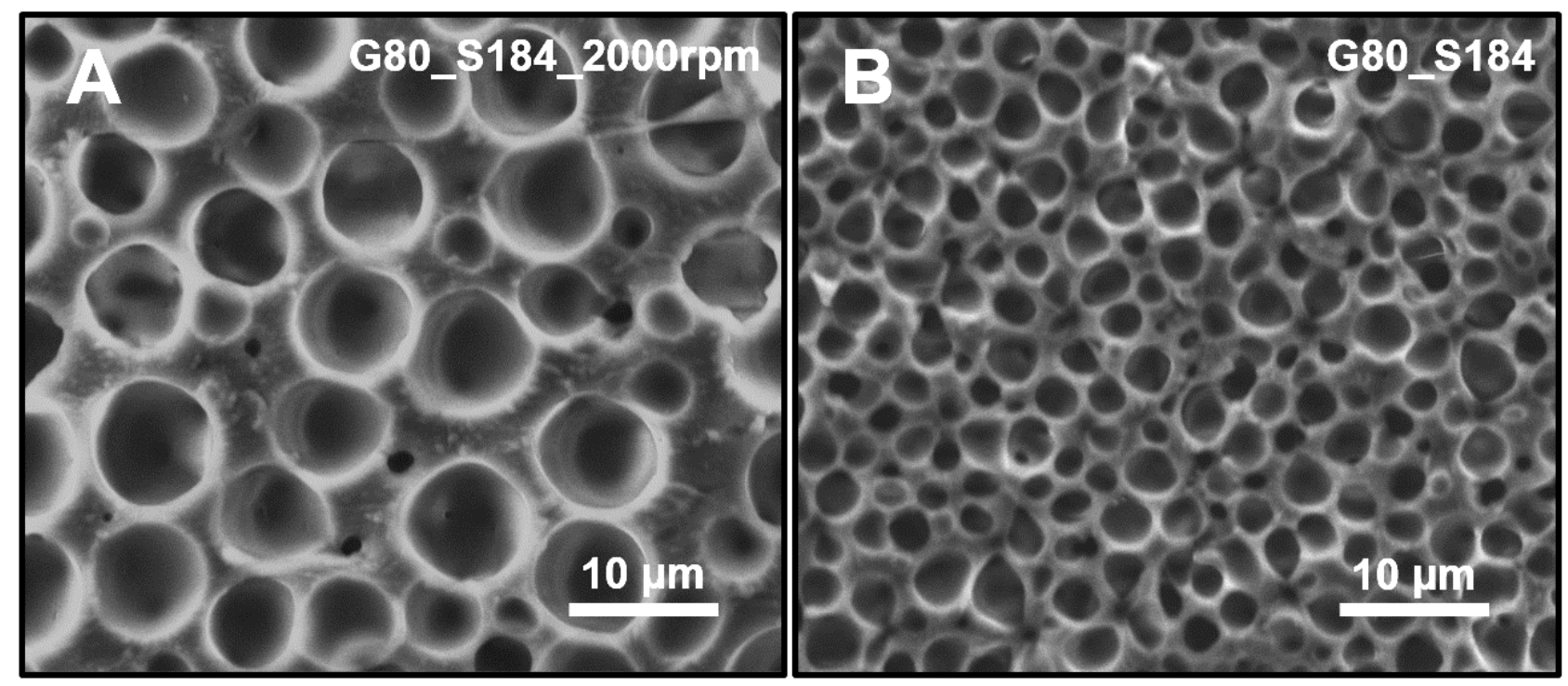

Figure 8. SEM images of composites based on G80_S184 formulation mixed with 2000 rpm (A) and $3500 \operatorname{rpm}(\mathrm{B})$.
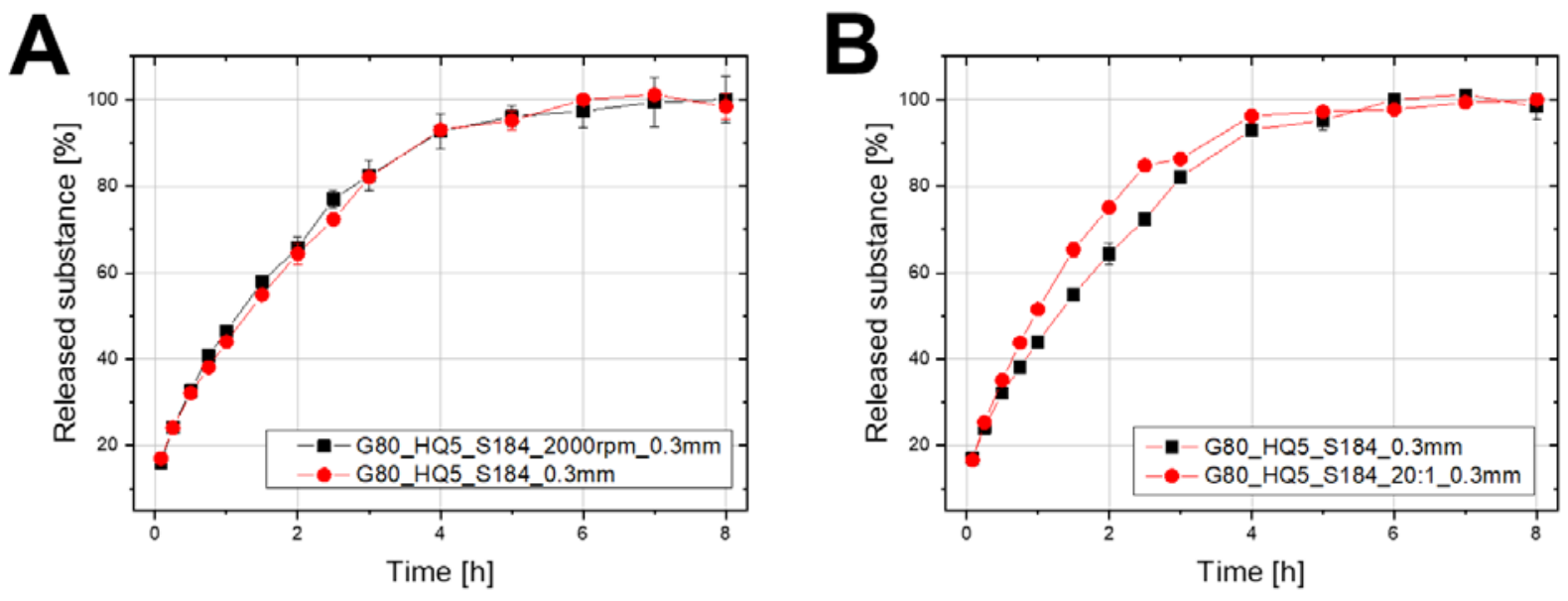

Figure 9. Release profiles of samples based on the formulation G80_HQ5_S184 prepared using different mixing speeds. 
Influence of mechanical properties on release

During the water absorption process the silicone phase of the composites with discrete glycerol domain morphology is stretched as the glycerol domains swell. Therefore, the silicone spacing between adjacent droplets becomes thinner facilitating faster transportation within the material and consequently faster release of substances from the material as concluded from the water absorption experiments discussed in a previous section. It was expected that the substance release rate from lowmodulus compositions would be accelerated compared to higher-modulus compositions, because of more facile water absorption. In this study the HQ release from the samples based on S184 mixed in different base:cross-linker ratios (10:1 and 20:1) and containing 80 phr of glycerol was investigated.

The release curves presented in Figure 9B clearly indicate that faster release of HQ occurred from the low-modulus composition. After 2.5 hours the high-modulus sample had released $72 \%$ of HQ while the low-modulus sample released $85 \%$. It is expected that it should be possible to more narrowly tune the release rate simply by modifying the structure of the elastomer.

The active substance release experiments clearly demonstrate that the drug delivery rate can be tuned simply by altering various formulation parameters during glycerol-silicone composite preparation. Depending on the glycerol loading zero-order, near zero-order and first-order release kinetics can be achieved. Additionally, the drug release rate can be adjusted by changing film thickness and mechanical properties of the silicone matrix.

The samples with the highest glycerol loadings are especially interesting as they represent a unique example of drug delivery technology exhibiting zero-order release behavior. Next to the exceptional release function the technology is biocompatible, majorly based on bio-based substrates and, foremost, it is cost-efficient, easy to implement and upscale. The glycerol-silicone elastomer 
matrices represent a novel family of two-phase elastomers that appears suitable for the wound care industry where smart functionalized materials are required.

\section{CONCLUSIONS}

Two-phase glycerol-silicone elastomeric composites with surprising functionality were prepared. Simple manipulation of the formulation allows one to control, at will, water absorption and substance release capabilities; the mechanical properties of the silicone elastomer can also be controlled. Most importantly, the matrices offer zero-order, near zero-order and first-order release behavior depending on the glycerol loading within the PDMS elastomer. Additionally, it was proven experimentally that by modifying various properties (mechanical properties or film thickness) the release rate of active substances can be precisely controlled.

\section{Acknowledgements}

The authors acknowledge the generous funding from Otto Mønsted Fonden for Prof Brook as a visiting professor at DTU.

\section{Supporting information}

1. Information about Sylgard 184. 2. Verification of the bicontinuous structure in the high loading glycerol-silicone elastomers. 3. Size and size distribution of glycerol droplets in the glycerol-silicone elastomers.

\section{References}


(1) Jain, K. K. Drug Delivery Systems; Humana Press: Basel, 2008.

(2) Schneider, C.; Langer, R.; Loveday, D.; Hair, D. Applications of Ethylene Vinyl Acetate Copolymers (EVA) in Drug Delivery Systems. J. Control. Release 2017, 262, 284-295.

(3) Chen, T.; Li, J.; Chen, T.; Sun, C. C.; Zheng, Y. Tablets of Multi-Unit Pellet System for Controlled Drug Delivery. J. Control. Release 2017, 262, 222-231.

(4) Peppas, N. A.; Khare, A. R. Preparation, Structure and Diffusional Behavior of Hydrogels in Controlled Release. Adv. Drug Deliv. Rev. 1993, 11, 1-35.

(5) Snorradóttir, B. S.; Jónsdóttir, F.; Sigurdsson, S. T.; Thorsteinsson, F.; Másson, M. Numerical Modelling and Experimental Investigation of Drug Release from Layered Silicone Matrix Systems. Eur. J. Pharm. Sci. 2013, 49, 671-678.

(6) Varelas, C. G.; Dixon, D. G.; Steiner, C. A. Zero-Order Release from Biphasic Polymer Hydrogels. J. Control. Release 1995, 34, 185-192.

(7) Bezemer, J. M.; Radersma, R.; Grijpma, D. W.; Dijkstra, P. J.; Feijen, J.; Van Blitterswijk, C. A. Zero-Order Release of Lysozyme from Poly(ethylene glycol)/Poly(butylene terephthalate) Matrices. J. Control. Release 2000, 64, 179-192.

(8) Xing, K.; Fan, R.; Wang, F.; Nie, H.; Du, X.; Gai, S.; Wang, P.; Yang, Y. Dual-StimulusTriggered Programmable Drug Release and Luminescent Ratiometric PH Sensing from Chemically Stable Biocompatible Zinc Metal-Organic Framework. ACS Appl. Mater. Interfaces 2018, 10, 22746-22756.

(9) Wang, Y.; Li, B.; Xu, F.; Han, Z.; Wei, D.; Jia, D.; Zhou, Y. Tough Magnetic Chitosan Hydrogel Nanocomposites for Remotely Stimulated Drug Release. Biomacromolecules 2018, 19, 3351-3360.

(10) Ruan, S.; Gu, Y.; Liu, B.; Gao, H.; Hu, X.; Hao, H.; Jin, L.; Cai, T. Long-Acting Release 
Microspheres Containing Novel GLP-1 Analog as an Antidiabetic System. Mol. Pharm. 2018, 15, 2857-2869.

(11) Ghaffari, R.; Eslahi, N.; Tamjid, E.; Simchi, A. Dual-Sensitive Hydrogel Nanoparticles Based on Conjugated Thermoresponsive Copolymers and Protein Filaments for Triggerable Drug Delivery. ACS Appl. Mater. Interfaces 2018, 10, 19336-19346.

(12) Gambles, M. T.; Fan, B.; Borecki, A.; Gillies, E. R. Hybrid Polyester Self-Immolative Polymer Nanoparticles for Controlled Drug Release. ACS Omega 2018, 3, 5002-5011.

(13) Wen, N.; Dong, Y.; Song, R.; Zhang, W.; Sun, C.; Zhuang, X.; Guan, Y.; Meng, Q.; Zhang, Y. Zero-Order Release of Gossypol Improves Its Antifertility Effect and Reduces Its Side Effects Simultaneously. Biomacromolecules 2018, 19, 1918-1925.

(14) Wang, J.; Cooper, R. C.; He, H.; Li, B.; Yang, H. Polyamidoamine Dendrimer Microgels: Hierarchical Arrangement of Dendrimers into Micrometer Domains with Expanded Structural Features for Programmable Drug Delivery and Release. Macromolecules 2018, 51, 61116118.

(15) Ferrati, S.; Fine, D.; You, J.; De Rosa, E.; Hudson, L.; Zabre, E.; Hosali, S.; Zhang, L.; Hickman, C.; Sunder Bansal, S.; Cordero-Reyes, A. M.; Genianatti, T.; Sih, J.; Goodall, R.; Palapattu, G.; Kloc, M.; Ghobrial, R. M.; Ferrari, M.; Grattoni, A. Leveraging Nanochannels for Universal, Zero-Order Drug Delivery in Vivo. J. Control. Release 2013, 172, 1011-1019.

(16) Feldstein, M. M.; Tohmakhchi, V. N.; Malkhazov, L. B.; Vasiliev, A. E.; Platé, N. A. Hydrophilic Polymeric Matrices for Enhanced Transdermal Drug Delivery. Int. J. Pharm. 1996, 131, 229-242.

(17) Jabbari, E.; Khakpour, M. Morphology of and Release Behavior from Porous Polyurethane Microspheres. Biomaterials 2000, 21, 2073-2079.

(18) Liu, H.; Farrell, S.; Uhrich, K. Drug Release Characteristics of Unimolecular Polymeric 
Micelles. J. Control. Release 2000, 68, 167-174.

(19) Matson, J. B.; Newcomb, C. J.; Bitton, R.; Stupp, S. I. Nanostructure-Templated Control of Drug Release from Peptide Amphiphile Nanofiber Gels. Soft Matter 2012, 8, 3586-3595.

(20) Guiseppi-Elie, A.; Brahim, S. I.; Recent, D. N. A Chemically Synthesized Artificial Pancreas : Adv. Mater. 2002, 14, 743-746.

(21) Fine, D.; Grattoni, A.; Hosali, S.; Ziemys, A.; De Rosa, E.; Gill, J.; Medema, R.; Hudson, L.; Kojic, M.; Milosevic, M.; Brousseau, L.; Goodall, R.; Ferrari, M.; Liu, X. A Robust Nanofluidic Membrane with Tunable Zero-Order Release for Implantable Dose Specific Drug Delivery. Lab Chip 2010, 10, 3074-3083.

(22) Woolfson, A. D.; Malcolm, R. K.; Gallagher, R. J. Design of a Silicone Reservoir Intravaginal Ring for the Delivery of Oxybutynin. J. Control. Release 2003, 91, 465-476.

(23) Malcolm, R. K.; McCullagh, S. D.; Woolfson, A. D.; Gorman, S. P.; Jones, D. S.; Cuddy, J. Controlled Release of a Model Antibacterial Drug from a Novel Self-Lubricating Silicone Biomaterial. J. Control. Release 2004, 97, 313-320.

(24) Murphy, P. S.; Evans, G. R. D. Advances in Wound Healing: A Review of Current Wound Healing Products. Plast. Surg. Int. 2012, 2012, 1-8.

(25) Silva, C. L.; Pereira, J. C.; Ramalho, A.; Pais, A. A. C. C.; Sousa, J. J. S. Films Based on Chitosan Polyelectrolyte Complexes for Skin Drug Delivery: Development and Characterization. J. Memb. Sci. 2008, 320, 268-279.

(26) Platt, A. J.; Phipps, A.; Judkins, K. A Comparative Study of Silicone Net Dressing and Paraffin Gauze Dressing in Skin-Grafted Sites. Burns 1996, 22, 543-545.

(27) Brook, M. A. Silicon in Organic, Organometallic, and Polymer Chemistry; John Wiley \& Sons Inc.: Ontario, 2000.

(28) Mojsiewicz-Pieńkowska, K.; Jamrógiewicz, M.; Zebrowska, M.; Mikolaszek, B.; Sznitowska, 
M. Double Layer Adhesive Silicone Dressing as a Potential Dermal Drug Delivery Film in Scar Treatment. Int. J. Pharm. 2015, 481, 18-26.

(29) Mazurek, P.; Hvilsted, S.; Skov, A. L. Green Silicone Elastomer Obtained from a Counterintuitively Stable Mixture of Glycerol and PDMS. Polymer 2016, 87, 1-7.

(30) Mazurek, P.; Yu, L.; Gerhard, R.; Wirges, W.; Skov, A. L. Glycerol as High-Permittivity Liquid Filler in Dielectric Silicone Elastomers. J. Appl. Polym. Sci. 2016, 133, 1-8.

(31) Lee, J. N.; Park, C.; Whitesides, G. M. Solvent Compatibility of Poly(dimethylsiloxane)-Based Microfluidic Devices. Anal. Chem. 2003, 75, 6544-6554.

(32) Larsen, A. L.; Hansen, K.; Sommer-Larsen, P.; Hassager, O.; Bach, A.; Ndoni, S.; Jørgensen, M. Elastic Properties of Nonstoichiometric Reacted PDMS Networks. Macromolecules 2003, 36, 10063-10070.

(33) Michalak, I.; Mucha, M. The Release of Active Substances from Selected Carbohydrate Biopolymer Membranes. Carbohydr. Polym. 2012, 87, 2432-2438.

(34) Gokhale, A. Achieving Zero-Order Release Kinetics Using Multi-Step Diffusion-Based Drug Delivery. PharmTech 2014, 38, 1-3.

(35) Liechty, W. B.; Kryscio, D. R.; Slaughter, B. V.; Peppas, N. A. Polymers for Drug Delivery Systems. Annu. Rev. Chem. Biomol. Eng. 2010, 1, 149-173. 


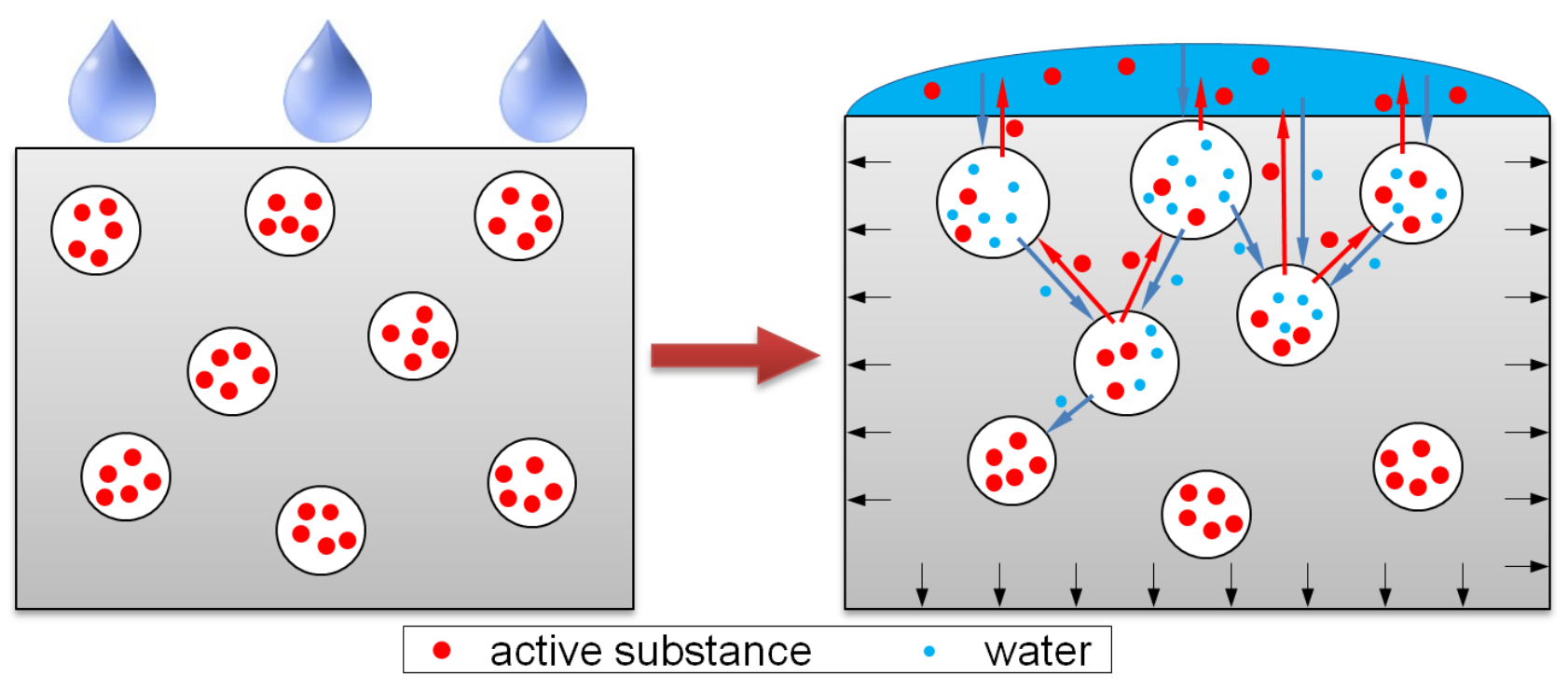

